\title{
VANISHING COTANGENT COHOMOLOGY FOR PLÜCKER ALGEBRAS
}

\author{
JAN ARTHUR CHRISTOPHERSEN AND NATHAN OWEN ILTEN
}

\begin{abstract}
We use representation theory and Bott's theorem to show vanishing of higher cotangent cohomology modules for the homogeneous coordinate ring of Grassmannians in the Plücker embedding. As a biproduct we answer a question of Wahl about the cohomology of the square of the ideal sheaf for the case of Plücker relations. We obtain slightly weaker vanishing results for the cotangent cohomology of the coordinate rings of isotropic Grassmannians.
\end{abstract}

\section{INTRODUCTION}

In this paper we prove the vanishing of cotangent cohomology $T_{A}^{i}$ for Plücker algebras $A$ when $i<\operatorname{dim} A$ and compute $T_{A}^{\operatorname{dim} A}$. We have also some (weaker) results for coordinate rings of isotropic Grassmannians. There are very few cases where higher cotangent cohomology has been computed, so we add some interesting examples to that short list.

Our main motivation though was to answer the following question when $X$ is a Grassmannian. Let $X=\operatorname{Proj} A$ be a projective variety with $Y$ a complete intersection in $X$. When is every deformation of $Y$ again a complete intersection in $X$ ? After imposing some mild hypotheses on $Y$, a sufficient condition for an affirmative answer consists of requiring the vanishing of certain higher cotangent cohomology modules of $A$. A precise statement is given in Proposition 5.2.

Fix a field $k$ of characteristic zero. If $\mathbb{G}=\mathbb{G}(r, n)$ is the Grassmannian of $r$-planes in an $n$ dimensional vector space over $k$, let $A$ be the corresponding Plücker algebra, i.e. $\mathbb{G}=\operatorname{Proj} A$ in the Plücker embedding. Set $d=r(n-r)+1$ to be the Krull dimension of $A$. Let $T_{A}^{i}=T^{i}(A / k ; A)$ denote the cotangent cohomology modules of $A$. We show that if $\mathbb{G} \neq \mathbb{G}(2,4)$ then $T_{A}^{i}=0$ for all $1 \leq i \leq d-1$. Moreover, $T_{A}^{d}=0$ if and only if $\mathbb{G}=\mathbb{G}(2, n)$ or $\mathbb{G}=\mathbb{G}(n-2, n)$. We give an example, $\mathbb{G}(2,6)$, where $T_{A}^{d+1} \neq 0$.

The case $T_{A}^{d}$ is of special interest since it is the vector space dual of $H_{\mathfrak{m}}^{0}\left(\Omega_{A}\right)$, where $\mathfrak{m}$ is the irrelevant maximal ideal and $\Omega_{A}$ is the module of Kähler differentials. The degree 2 part of this is isomorphic to the kernel of the Gaussian map

$$
\bigwedge^{2} H^{0}\left(\mathbb{G}, \mathcal{O}_{\mathbb{G}}(1)\right) \rightarrow H^{0}\left(\mathbb{G}, \Omega_{\mathbb{G}} \otimes \mathcal{O}_{\mathbb{G}}(1)^{2}\right)
$$

where $\mathcal{O}_{\mathbb{G}}(1)$ is for the Plücker embedding. We show that the graded pieces $H_{\mathfrak{m}}^{0}\left(\Omega_{A}\right)_{m}=0$ for $m \neq 2$, which is an affirmative answer to a question by Jonathan Wahl in the case $G / P$ is a Grassmannian. See Theorem 3.6 and the following remark.

Since $A$ is Cohen-Macaulay and $\operatorname{Spec} A$ has one singular point at $\mathfrak{m}$ we have isomorphisms $T_{A}^{i} \simeq \operatorname{Ext}_{A}^{i}\left(\Omega_{A}, A\right)$. Because of the isolated singularity we furthermore get $\operatorname{Ext}_{A}^{i}\left(\Omega_{A}, A\right) \simeq$

Date: October 13, 2016.

2000 Mathematics Subject Classification. Primary 14M15; Secondary 14B15, 14D15, 13D03.

Key words and phrases. Grassmannians. Cotangent cohomology. Local cohomology. 
$H_{\mathfrak{m}}^{i+1}\left(\operatorname{Der}_{k}(A)\right)$ for $1 \leq i \leq d-2$. In general, the vanishing of these local cohomology modules in the case $X=\operatorname{Proj} A$ is smooth is related to cohomology vanishing for twists of $\mathcal{O}_{X}$ and $\Theta_{X}$. Thus vanishing of $T_{A}^{i}$ in the range $1 \leq i \leq d-2$ may be shown by proving vanishing of $H^{i}\left(X, \mathcal{O}_{X}(m)\right)$ and $H^{i}\left(X, \Theta_{X}(m)\right)$, a result originally shown by Svanes. For our $\mathbb{G}(r, n)$ we use Bott's theorem and an argument involving the Atiyah extension to show $H_{\mathfrak{m}}^{i+1}\left(\operatorname{Der}_{k}(A)\right)=0$ for $1 \leq i \leq d-2$. See Section 3.1.

For the remaining two cases, by local duality we have $T_{A}^{d-i} \simeq H_{\mathfrak{m}}^{i}\left(\Omega_{A}\right)^{*}$ for $i=0,1$. Here $M^{*}$ denotes the $k$-dual. If $G=\mathrm{SL}_{r}$ and $S=k\left[x_{i j}: 1 \leq i \leq n, 1 \leq j \leq r\right]$ is the ring of functions on the vector space of $n \times r$ matrices, then $A=S^{G}$. In Section 2.3 we consider the general situation where $S$ is a finitely generated standard graded $k$-algebra with the action of a linearly reductive group $G$ respecting the grading.

We must assume that $\operatorname{Spec} S^{G}$ has an isolated singularity at the irrelevant maximal ideal $\mathfrak{m} \subset S^{G}$ and that both $\operatorname{depth}_{\mathfrak{m} S} S \geq 2$ and $\operatorname{depth}_{\mathfrak{m} S} \Omega_{S} \geq 2$. Under these conditions we exhibit a four term complex of free $S[G]$-modules, which after taking invariants computes $H_{\mathfrak{m}}^{i}\left(\Omega_{S^{G}}\right)$ for $i=0,1$. This allows us to use representation theory to compute the local cohomology. We do this for our case using the combinatorics of Schur functors in Section 3.2.

In the case of isotropic Grassmannians, we also understand enough about the tangent sheaf to apply Bott's theorem to get results similar to above, see Section 4 . Indeed, let $A$ be the coordinate ring of an isotropic Grassmannian $X$ in its Plücker embedding, not equal to the symplectic Grassmannian $\mathbb{L} \mathbb{G}(3,6)$ of 3 -planes in a 6 -dimensional vector space. Then $T_{A}^{i}=0$ for all $2 \leq i \leq d-3$. We show that $T_{A}^{d-2}=0$ if and only if $X$ is $\mathbb{L} \mathbb{G}(n-1,2 n)$ or $\mathbb{O} \mathbb{G}(n, 2 n+1)$. Furthermore, $T_{A}^{1}=0$ as long as $X$ is not an isotropic Grassmannian of 1 or 2-planes, or $\mathbb{O} \mathbb{G}(4,8)$.

This work was motivated by our attempt to understand the smoothings of certain degenerate Fano varieties in homogeneous spaces. In our last Section we give an application regarding deformations of complete intersections in cones over Grassmannians.

Acknowledgments. We would like to thank Steven Sam for helpful discussions on representation theory.

\section{Preliminaries}

1.1. Cotangent cohomology. To fix notation we give a short description of the cotangent modules and sheaves. For definitions, proofs and details on this cohomology and its relevance to deformation theory see [And74], [Ill71] and [Lau79]. Given a ring $R$ and an $R$-algebra $S$ there is a complex of free $S$ modules; the cotangent complex $\mathbb{L}_{\bullet}^{S / R}$. See e.g. [And74, p. 34] for a definition.

For an $S$ module $M$ we get the cotangent cohomology modules

$$
T^{i}(S / R ; M)=H^{i}\left(\operatorname{Hom}_{S}\left(\mathbb{L}_{\bullet}^{S / R}, M\right)\right)
$$

and cotangent homology modules

$$
T_{i}(S / R ; M)=H_{i}\left(\mathbb{L}_{\bullet}^{S / R} \otimes_{S} M\right) .
$$

If $R$ is the ground field we abbreviate $T^{i}(S / R ; M)=T_{S}^{i}(M)$ and $T_{S}^{i}(S)=T_{S}^{i}$. Correspondingly we will write $T_{i}^{S / R}$ for the homology. There is a natural spectral sequence

$$
\operatorname{Ext}_{S}^{p}\left(T_{q}^{S / R}, M\right) \Rightarrow T^{p+q}(S / R ; M)
$$

which will in our case allow us to compute $T_{A}^{i}$ as $\operatorname{Ext}_{A}^{i}\left(\Omega_{A}, A\right)$. See Proposition 2.1. 
1.2. Representation theory. We review our notation and some theory which we have taken from [FH91], [Wey03] and [RWW14]. A weight of the maximal torus of diagonal matrices in $\mathrm{GL}_{n}$ is an $n$-tuple $\lambda=\left(\lambda_{1}, \ldots, \lambda_{n}\right) \in \mathbb{Z}^{n}$. It is dominant if $\lambda_{1} \geq \lambda_{2} \geq \cdots \geq \lambda_{n}$. We will often use the shorthand $\lambda=\left(n_{1}^{a_{1}}, \ldots, n_{k}^{a_{k}}\right)$ meaning $n_{i}$ is repeated $a_{i}$ times in the tuple. If $\lambda$ is a dominant weight with $\lambda_{n} \geq 0$ then $\lambda$ yields a partition of $m=\sum \lambda_{i}$ and we denote this $\lambda \vdash m$. If it is clear that $\lambda$ is a partition then we do not include the trailing zeros in the tuple.

Given an $n$-dimensional vector space $E$ the irreducible representations of $\mathrm{GL}_{n} \simeq \mathrm{GL}(E)$ are in one-to-one correspondence with the dominant weights. We write $\mathbb{S}_{\lambda} E$ for the corresponding Schur functor, i.e. the irreducible representation associated to $\lambda$. We have $\mathbb{S}_{\left(1^{r}\right)} E=\wedge^{r} E$, $\mathbb{S}_{\lambda} E \otimes \wedge^{n} E=\mathbb{S}_{\lambda+\left(1^{n}\right)}$ and $\mathbb{S}_{\lambda} E^{*}=\mathbb{S}_{\left(-\lambda_{n}, \ldots,-\lambda_{1}\right)} E$. If $E$ and $F$ are vector spaces we have the Cauchy formula for $\operatorname{Sym}^{k}(E \otimes F)$ as $\mathrm{GL}(E) \times \mathrm{GL}(F)$-representation, namely

$$
\operatorname{Sym}^{k}(E \otimes F)=\bigoplus_{\lambda \vdash k} \mathbb{S}_{\lambda} E \otimes \mathbb{S}_{\lambda} F
$$

This and several other standard combinatorial statements (which may be found in the above mentioned literature) relating to the Littlewood-Richardson rule and Young diagrams are used in Section 3.2.

1.3. Bott's theorem for the Grassmannian. Let $\mathbb{G}=\mathbb{G}(r, E)$ be the Grassmannian of $r$-dimensional subspaces of $E$ and let

$$
0 \rightarrow \mathcal{R} \rightarrow \mathcal{O}_{\mathbb{G}} \otimes E \rightarrow \mathcal{Q} \rightarrow 0
$$

be the tautological sequence on $\mathbb{G}$. By functoriality the Schur functors may be applied to vector bundles on the $\mathbb{G}$, in particular to the tautological sub and quotient bundles $\mathcal{R}$ and $\mathcal{Q}$.

We review Bott's theorem applied to $\mathbb{G}$ as described in [RWW14, Section 2.2]. It will be used in Section 3.1. Consider two dominant weights $\alpha=\left(\alpha_{1}, \ldots, \alpha_{n-r}\right)$ and $\beta=\left(\beta_{1}, \ldots, \beta_{r}\right)$ and their concatenation $\gamma=\left(\gamma_{1}, \ldots, \gamma_{n}\right)$. Let $\delta=(n-1, \ldots, 0)$ and consider $\gamma+\delta$. Write $\operatorname{sort}(\gamma+\delta)$ for the sequence obtained by arranging the entries of $\gamma+\delta$ in non-increasing order, and define $\tilde{\gamma}=\operatorname{sort}(\gamma+\delta)-\delta$.

Theorem 1.1 (Bott). With the above notation, if $\gamma+\delta$ has repeated entries, then

$$
H^{i}\left(\mathbb{G}, \mathbb{S}_{\alpha} \mathcal{Q} \otimes \mathbb{S}_{\beta} \mathcal{R}\right)=0
$$

for all $i \geq 0$. Otherwise, writing $l$ for the number of pairs $(i, j)$ with $1 \leq i<j \leq n$ and $\gamma_{i}-i<\gamma_{j}-j$, we have

$$
H^{l}\left(\mathbb{G}, \mathbb{S}_{\alpha} \mathcal{Q} \otimes \mathbb{S}_{\beta} \mathcal{R}\right)=\mathbb{S}_{\tilde{\gamma}} E
$$

and $H^{i}\left(\mathbb{G}, \mathbb{S}_{\alpha} \mathcal{Q} \otimes \mathbb{S}_{\beta} \mathcal{R}\right)=0$ for $i \neq l$.

We will also apply Bott's theorem to isotropic Grassmannians in Section 4. We refer the reader to [Wey03, 4.3] for details.

\section{Computing higher cotangent Cohomology}

We give here in successively more special cases the methods we will use to compute the higher $T^{i}$. 


\subsection{Cohen-Macaulay isolated singularities.}

Proposition 2.1. Let $(A, \mathfrak{m})$ be a d-dimensional Cohen-Macaulay local $k$-algebra such that $\operatorname{Spec} A$ is an isolated singularity. Then

$$
T_{A}^{i} \simeq \operatorname{Ext}_{A}^{i}\left(\Omega_{A}, A\right)
$$

for $0 \leq i \leq d$.

Proof. Consider the spectral sequence $\operatorname{Ext}_{A}^{p}\left(T_{q}^{A}, A\right) \Rightarrow T_{A}^{n}$ and note that by the depth condition $\operatorname{Ext}_{A}^{p}\left(T_{q}^{A}, A\right)$ vanishes if $q \geq 1$ and $p<d$.

Lemma 2.2. Let $(A, \mathfrak{m})$ be a d-dimensional Cohen-Macaulay local $k$-algebra such that Spec $A$ is an isolated singularity. Then

$$
\operatorname{Ext}_{A}^{i}\left(\Omega_{A}, A\right) \simeq H_{\mathfrak{m}}^{i+1}\left(\operatorname{Der}_{k}(A)\right)
$$

for $1 \leq i \leq d-2$.

Proof. We will use Ext with support as described in SGA 2 Exposè VI ([Gro05]), specifically $\operatorname{Ext}_{\mathfrak{m}}^{i}\left(\Omega_{A}, A\right)$. Consider first the spectral sequence

$$
\operatorname{Ext}_{A}^{p}\left(\Omega_{A}, H_{\mathfrak{m}}^{q}(A)\right) \Rightarrow \operatorname{Ext}_{\mathfrak{m}}^{n}\left(\Omega_{A}, A\right)
$$

which shows that $\operatorname{Ext}_{\mathfrak{m}}^{i}\left(\Omega_{A}, A\right)=0$ for $i<d$. If $U=\operatorname{Spec} A \backslash V(\mathfrak{m})$, there is a long exact sequence

$$
\cdots \rightarrow \operatorname{Ext}_{\mathfrak{m}}^{i}\left(\Omega_{A}, A\right) \rightarrow \operatorname{Ext}_{A}^{i}\left(\Omega_{A}, A\right) \rightarrow \operatorname{Ext}_{U}^{i}\left(\Omega_{U}, \mathcal{O}_{U}\right) \rightarrow \operatorname{Ext}_{\mathfrak{m}}^{i+1}\left(\Omega_{A}, A\right) \rightarrow \cdots
$$

and it follows that $\operatorname{Ext}_{A}^{i}\left(\Omega_{A}, A\right) \simeq \operatorname{Ext}_{U}^{i}\left(\Omega_{U}, \mathcal{O}_{U}\right)$ for $i \leq d-2$. On the other hand $\operatorname{Ext}_{U}^{i}\left(\Omega_{U}, \mathcal{O}_{U}\right) \simeq H^{i}\left(U, \Theta_{U}\right)$ which again is isomorphic to $H_{\mathfrak{m}}^{i+1}\left(\operatorname{Der}_{k}(A)\right)$ for $i \geq 1$.

Proposition 2.3. Let $(A, \mathfrak{m})$ be a d-dimensional Cohen-Macaulay local $k$-algebra with $d \geq 2$, such that $\operatorname{Spec} A$ is an isolated singularity. Then

$$
T_{A}^{i} \simeq H_{\mathfrak{m}}^{i+1}\left(\operatorname{Der}_{k}(A)\right) \quad \text { if } 1 \leq i \leq d-2 .
$$

If moreover $(A, \mathfrak{m})$ is Gorenstein then $T_{A}^{d-1} \simeq H_{\mathfrak{m}}^{1}\left(\Omega_{A}\right)^{*}$ and $T_{A}^{d} \simeq H_{\mathfrak{m}}^{0}\left(\Omega_{A}\right)^{*}$.

Proof. This follows directly from Proposition 2.1, Lemma 2.2 and local duality.

2.2. Computing $H_{\mathfrak{m}}^{i}\left(\operatorname{Der}_{k}(A)\right)$ for cones over projective schemes. Some of the ideas in this section were used by Svanes and Schlessinger and may be found in [Sva75] and [Sch71]. We believe our approach is more direct and gives more than the vanishing of the cohomology. To use Proposition 2.3 we need to compute the local cohomology of the derivation module. For cones over projective schemes $X$ we may relate this to the sheaf cohomology of twists of $\mathcal{O}_{X}$ and $\Theta_{X}$.

Let $A$ be a standard graded $k$-algebra, i.e. the algebra generators are in degree 1. Let $X=\operatorname{Proj} A$ with irrelevant maximal ideal $\mathfrak{m}$. Let $X^{\prime}=\operatorname{Spec} A \backslash V(\mathfrak{m}), \pi: X^{\prime} \rightarrow X$ the $\mathbb{G}_{m}$ quotient and set $\mathcal{S}=\pi_{*} \mathcal{O}_{X^{\prime}}$ a sheaf of graded algebras on $X$ with $\mathcal{S}_{0}=\mathcal{O}_{X}$. Let $\Theta_{\mathcal{S}}$ the sheaf which is locally $\operatorname{Der}_{k}(\mathcal{S}(U))$ for open affine $U \subset X$, i.e. $\Theta_{\mathcal{S}}=\pi_{*} \Theta_{X^{\prime}}$. Then $\Theta_{\mathcal{S}}$ is a sheaf of graded $\mathcal{S}$-modules so let $\mathcal{E}$ be the degree 0 part.

If $\mathcal{S}(U)=B$, so that $\mathcal{O}_{X}(U)=B_{0}$ then the sequence

$$
0 \rightarrow \operatorname{Der}_{B_{0}}(B) \rightarrow \operatorname{Der}_{k}(B) \rightarrow \operatorname{Der}_{k}\left(B_{0}, B\right) \rightarrow 0
$$


is exact since $B$ is smooth over $B_{0}$. Moreover the Euler derivation gives a graded isomorphism $B \simeq \operatorname{Der}_{B_{0}}(B)$. This globalizes to an exact sequence

$$
0 \rightarrow \mathcal{S} \rightarrow \Theta_{\mathcal{S}} \rightarrow \Theta_{X} \otimes_{\mathcal{O}_{X}} \mathcal{S} \rightarrow 0
$$

and taking the degree 0 part we get

$$
0 \rightarrow \mathcal{O}_{X} \rightarrow \mathcal{E} \rightarrow \Theta_{X} \rightarrow 0
$$

This sequence is locally

$$
0 \rightarrow B_{0} \rightarrow \operatorname{Der}_{k}(B)_{0} \rightarrow \operatorname{Der}_{k}\left(B_{0}\right) \rightarrow 0
$$

so we see that $\mathcal{E} \simeq \widetilde{\operatorname{Der}_{k}(A)}$. Recall that by comparing the Čech complex of $\widetilde{M}$ over Proj $A$ and the complex computing $H_{\mathfrak{m}}^{i}(M)$ we get $\bigoplus_{m} H^{i}(\operatorname{Proj} A, \widetilde{M}(m)) \simeq H_{\mathfrak{m}}^{i+1}(M)$ when $i \geq 1$. Thus we have proven

Lemma 2.4. There are isomorphisms $H_{\mathfrak{m}}^{i}\left(\operatorname{Der}_{k}(A)\right) \simeq \bigoplus_{m \in \mathbb{Z}} H^{i-1}(X, \mathcal{E}(m))$ for $i \geq 2$.

Proposition 2.5 ([Sva75] Remark 2.5). Assume $X=\operatorname{Proj} A$ is smooth and the local ring $(A, \mathfrak{m})$ is Cohen-Macaulay. Let $1 \leq j \leq \operatorname{dim} X-1$. If

$$
H^{i}\left(X, \mathcal{O}_{X}(m)\right)=H^{i}\left(X, \Theta_{X}(m)\right)=0
$$

for all $m$ and all $1 \leq i \leq j$, then $T_{A}^{i}=0$ for all $1 \leq i \leq j$.

Proof. This follows from Proposition 2.3, the exact sequence (2.1) and Lemma 2.4.

We will need to prove the vanishing of $T_{A}^{d-2}$ even though not all $H^{d-2}\left(X, \Theta_{X}(m)\right)=0$. For this we need to understand the sequence (2.1) better. For any scheme there is a natural map $\mathcal{O}_{X}^{*} \rightarrow \Omega_{X}$ defined locally by

$$
u \mapsto \frac{d u}{u}
$$

Let $c: H^{1}\left(X, \mathcal{O}_{X}^{*}\right) \rightarrow H^{1}\left(X, \Omega_{X}\right)$ be the induced map in cohomology. Now $H^{1}\left(X, \Omega_{X}\right) \simeq$ $\operatorname{Ext}^{1}\left(\mathcal{O}_{X}, \Omega_{X}\right)$, so for a line bundle $L, c(L)$ gives an extension

$$
e_{L}: \quad 0 \rightarrow \Omega_{X} \rightarrow \mathcal{F}_{L} \rightarrow \mathcal{O}_{X} \rightarrow 0 .
$$

Set $\mathcal{E}_{L}:=\mathcal{F}_{L}^{\vee}$ and note that the dual sequence

$$
e_{L}^{\vee}: \quad 0 \rightarrow \mathcal{O}_{X} \rightarrow \mathcal{E}_{L} \rightarrow \Theta_{X} \rightarrow 0
$$

is also exact since $\mathcal{O}_{X}$ is the sheaf on the right. In the smooth case this is known as the Atiyah extension associated to $L$, but we will call it that for general $X$.

We state and prove for lack of reference (in this generality) the certainly well known

Proposition 2.6. If $X=\operatorname{Proj} A$ and $L=\mathcal{O}_{X}(1)=\widetilde{A(1)}$ then the sequence

$$
0 \rightarrow \mathcal{O}_{X} \rightarrow \mathcal{E} \rightarrow \Theta_{X} \rightarrow 0
$$

is the Atiyah extension $e_{L}^{\vee}$.

Proof. Let $x_{0}, \ldots x_{n}$ be a basis for $H^{0}\left(X, \mathcal{O}_{X}(1)\right)$ so we may realize $X$ in $\mathbb{P}^{n}$. Set $B=A_{\left(x_{0}\right)}=$ $k\left[x_{0}, \ldots, x_{n}, x_{0}^{-1}\right] / I$ for some ideal $I$. Then $B_{0}=k\left[y_{1}, \ldots, y_{n}\right] / J$ where $J$ is generated by the $f\left(1, y_{1}, \ldots, y_{n}\right)$ with $f \in I$ and the inclusion is given by $y_{i} \mapsto x_{i} x_{0}^{-1}$. For a homogeneous $f \in B_{d}$

$$
f\left(x_{0}, \ldots, x_{n}\right)=x_{0}^{d} f\left(1, \frac{x_{1}}{x_{0}}, \ldots, \frac{x_{n}}{x_{0}}\right)
$$


Write $\partial_{x}$ for the partial derivative of a variable $x$. A derivation $D \in \operatorname{Der}\left(B_{0}\right)$ can be written $D=\sum_{i} a_{i} \partial_{y_{i}}$ where the $a_{i}$ are such that $D(f)=0$ in $B_{0}$ for all $f \in J$. From $D$ we can form

$$
\widetilde{D}=\sum_{i=1}^{n} a_{i}\left(\frac{x_{1}}{x_{0}}, \ldots, \frac{x_{n}}{x_{0}}\right) \cdot x_{0} \partial_{x_{i}} .
$$

Using (2.2) one can check that $\widetilde{D}$ is a well defined derivation of $B$. It is clearly of degree 0 . Moreover one may compute that for $D \in \operatorname{Der}(B)_{0}$

$$
D-\widetilde{D_{\mid B_{0}}}=g \sum_{i=0}^{n} x_{i} \partial_{x_{i}}
$$

for suitable $g$. This implies that (2.1) is locally split.

The sequence $e_{L}^{\vee}$ is also locally split and we may write $\mathcal{E}_{L}$ locally on $U_{i}$ as $\mathcal{O}_{U_{i}} \oplus \Theta_{U_{i}}$. Let $L$ be represented by a Čech cocycle $\left(f_{i j}\right), f_{i j} \in \Gamma\left(U_{i j}, \mathcal{O}_{X}^{*}\right)$. The gluing of $\mathcal{E}_{L}$ is determined (dually) by the extension class in $H^{1}\left(\Omega_{X}\right) ;\left(g_{i}, D_{i}\right) \in \Gamma\left(U_{i}, \mathcal{E}_{L}\right)$ and $\left(g_{j}, D_{j}\right) \in \Gamma\left(U_{j}, \mathcal{E}_{L}\right)$ are equal on $U_{i j}$ iff $D_{i}=D_{j}$ and $g_{j}-g_{i}=D_{i}\left(f_{i j}\right) / f_{i j}$. Now use the above local splitting to show that when $L=\mathcal{O}_{X}(1)$ we have $\mathcal{E} \simeq \mathcal{E}_{L}$.

2.3. Computing $H_{\mathfrak{m}}^{0}\left(\Omega_{S^{G}}\right)$ and $H_{\mathfrak{m}}^{1}\left(\Omega_{S^{G}}\right)$ for invariant rings. Let $S$ be a finitely generated standard graded $k$-algebra with the action of a linearly reductive group $G$ respecting the grading. Assume Spec $S^{G}$ has an isolated singularity at $\mathfrak{m} \subset S^{G}$. If $J=\mathfrak{m} S$ assume that $\operatorname{depth}_{J} S \geq 2$ and that $\operatorname{depth}_{J} \Omega_{S} \geq 2$.

Let $H$ be the kernel of the map $\Omega_{S^{G}} \otimes_{S^{G}} S \rightarrow \Omega_{S}$.

Lemma 2.7. There are isomorphisms

$$
H_{\mathfrak{m}}^{0}\left(\Omega_{S^{G}}\right) \simeq H^{G} \quad \text { and } \quad H_{\mathfrak{m}}^{1}\left(\Omega_{S^{G}}\right) \simeq H_{J}^{0}\left(\Omega_{S / S^{G}}\right)^{G} .
$$

Proof. Consider the exact sequence

$$
0 \rightarrow H \rightarrow \Omega_{S^{G}} \otimes_{S^{G}} S \rightarrow \Omega_{S} \rightarrow \Omega_{S / S^{G}} \rightarrow 0
$$

and note that $\left(\Omega_{S^{G}} \otimes_{S^{G}} S\right)^{G}=\Omega_{S^{G}}$. We split the sequence into 2 short exact sequences. On the right we get

$$
0 \rightarrow K \rightarrow \Omega_{S} \rightarrow \Omega_{S / S^{G}} \rightarrow 0
$$

which yields $H_{J}^{0}(K)=0$ and $H_{J}^{1}(K) \simeq H_{J}^{0}\left(\Omega_{S / S^{G}}\right)$. On the left we get

$$
0 \rightarrow H \rightarrow \Omega_{S^{G}} \otimes_{S^{G}} S \rightarrow K \rightarrow 0 .
$$

The module $H$ is supported at $J$ so $H_{J}^{i}(H)=0$ for $i \geq 1$ and the sequence yields $H \simeq$ $H_{J}^{0}(H) \simeq H_{J}^{0}\left(\Omega_{S^{G}} \otimes_{S^{G}} S\right)$ and $H_{J}^{0}\left(\Omega_{S / S^{G}}\right) \simeq H_{J}^{1}(K) \simeq H_{J}^{1}\left(\Omega_{S^{G}} \otimes_{S^{G}} S\right)$. Taking invariants yields the result.

A series of right exact sequences

$$
B^{i-1} \stackrel{\beta_{i-1}}{\longrightarrow} C^{i} \stackrel{\gamma_{i}}{\longrightarrow} B^{i} \rightarrow 0
$$

leads to a complex

$$
\cdots \stackrel{\delta_{i-2}}{\longrightarrow} C^{i-1} \stackrel{\delta_{i-1}}{\longrightarrow} C^{i} \stackrel{\delta_{i}}{\longrightarrow} C^{i+1} \stackrel{\delta_{i+1}}{\longrightarrow} \cdots
$$

with $\delta_{i}=\beta_{i} \circ \gamma_{i}$. Moreover since the sequences are right exact we have $H^{i}\left(C^{\bullet}\right) \simeq \operatorname{Ker} \beta_{i}$. We will use this construction to get a four term complex which computes the local cohomology we are interested in. 
Let $\mathfrak{g}$ be the Lie algebra of $G$. By [CK14, Lemma 4.7] there are isomorphisms

$$
\operatorname{Hom}_{S}\left(\Omega_{S / S^{G}}, S\right) \simeq \operatorname{Der}_{S^{G}}(S) \simeq S \otimes \mathfrak{g} .
$$

Choosing a basis for $\mathfrak{g}$ defines a $G$-equivariant map $\Omega_{S / S^{G}} \stackrel{E}{\rightarrow} S \otimes \mathfrak{g}^{*} \simeq j_{*} j^{*} \Omega_{S / S^{G}}$ where $j$ is the inclusion of Spec $S \backslash V(J)$ in $\operatorname{Spec} S$ (see [CK14, Section 4.2]). Thus we have an exact sequence

$$
0 \rightarrow H_{J}^{0}\left(\Omega_{S / S^{G}}\right) \rightarrow \Omega_{S / S^{G}} \stackrel{E}{\rightarrow} S \otimes \mathfrak{g}^{*} \rightarrow H_{J}^{1}\left(\Omega_{S / S^{G}}\right) \rightarrow 0 .
$$

Assume that the algebra generators of $S^{G}$ are in a single degree in $S$, i.e. that they generate a subspace $U^{*}$ of a certain $S_{r}$. The invariant polynomials define an embedding $\operatorname{Spec} S^{G} \subset U$.

Set $P=\operatorname{Sym} U^{*}$ and let $I$ be the kernel of $P \rightarrow S^{G}$. Assume that the generators of $I$ are in a single degree and span a subspace $F \subseteq P_{s}$. Now $\Omega_{P} \otimes_{P} S^{G} \otimes_{S G} S \simeq P \otimes_{k} U^{*} \otimes_{P} S \simeq S \otimes_{k} U^{*}$ and $I / I^{2} \otimes_{S^{G}} S \simeq I \otimes_{P} S$ is the image of $S \otimes F$ so we get an exact sequence

$$
S \otimes F \rightarrow S \otimes U^{*} \rightarrow \Omega_{S^{G}} \otimes_{S^{G}} S \rightarrow 0 .
$$

We construct our complex from the right parts of the sequences (2.3) and (2.4) together with (2.5). We put everything into a diagram with exact rows and columns. The complex then consists of the diagonal maps in

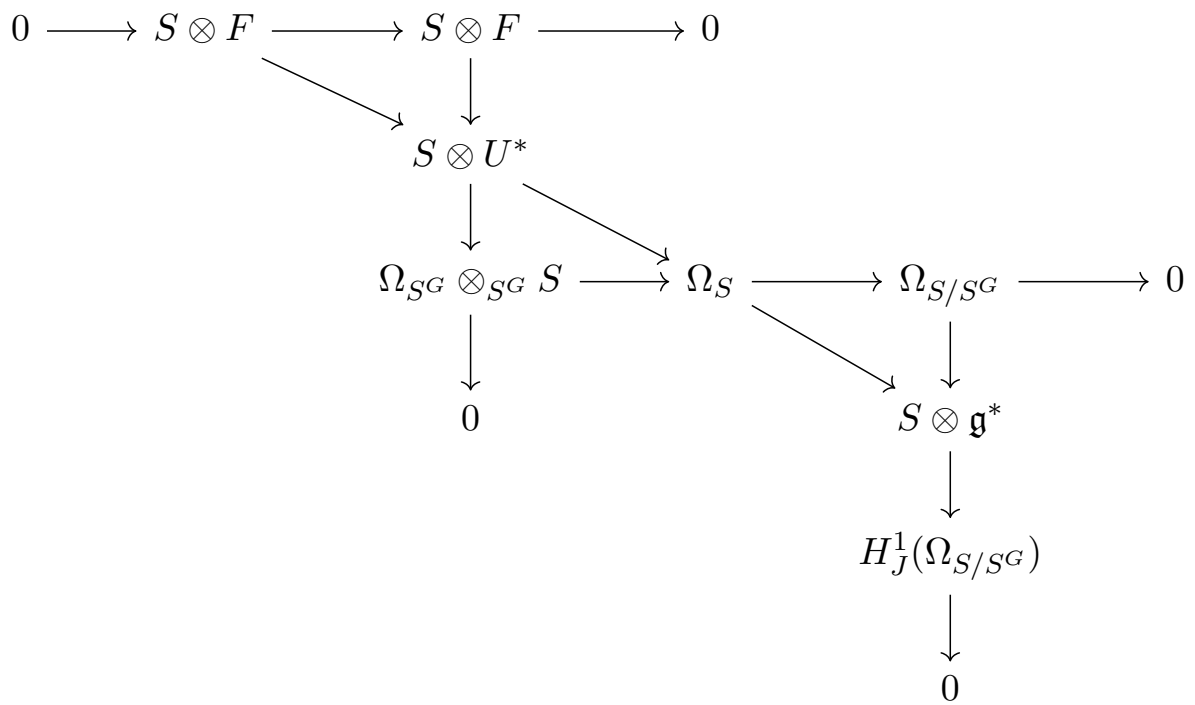

so we have proven

Proposition 2.8. The four term complex

$$
C^{\bullet}: \quad(S \otimes F)^{G} \stackrel{d^{1}}{\rightarrow}\left(S \otimes U^{*}\right)^{G} \stackrel{d^{2}}{\longrightarrow}\left(\Omega_{S}\right)^{G} \stackrel{d^{3}}{\longrightarrow}\left(S \otimes \mathfrak{g}^{*}\right)^{G}
$$

has $H^{1}\left(C^{\bullet}\right) \simeq H_{\mathfrak{m}}^{0}\left(\Omega_{S^{G}}\right)$ and $H^{2}\left(C^{\bullet}\right) \simeq H_{\mathfrak{m}}^{1}\left(\Omega_{S^{G}}\right)$.

To apply this we will need a more detailed description of $d^{3}$ in the case when $S=\operatorname{Sym} V^{*}$ for a $G$-representation $V$. Let $x_{1}, \ldots, x_{n}$ be a basis for $\operatorname{Sym}^{1} V^{*}$. We start with the dual cotangent sequence for $k \rightarrow S^{G} \rightarrow S$, i.e.

$$
0 \rightarrow \operatorname{Der}_{S^{G}}(S) \rightarrow \operatorname{Der}_{k}(S) \rightarrow \operatorname{Der}_{k}\left(S^{G}, S\right)
$$


which under the assumptions is also right exact (see e.g. [CK14, Section 4.2]). We have (see above) $\operatorname{Der}_{S^{G}}(S) \simeq S \otimes \mathfrak{g}$ and we always have $\operatorname{Der}_{k}(S) \simeq S \otimes_{k} V$ using $\frac{\partial}{\partial x_{i}}$ as a basis for $V$.

Let

$$
\rho: \mathfrak{g} \rightarrow \operatorname{Sym}^{1} V^{*} \otimes V \simeq \operatorname{Hom}(V, V)
$$

be the induced representation of the Lie algebra. On graded pieces we have

$$
S \otimes \mathfrak{g} \simeq \operatorname{Der}_{S^{G}}(S) \rightarrow \operatorname{Der}_{k}(S) \simeq S \otimes V
$$

given by the composite

$$
\mathrm{Sym}^{k} V^{*} \otimes \mathfrak{g} \stackrel{\text { id } \otimes \rho}{\longrightarrow} \operatorname{Sym}^{k} V^{*} \otimes \mathrm{Sym}^{1} V^{*} \otimes V \stackrel{\mu \otimes \mathrm{id}}{\longrightarrow} \mathrm{Sym}^{k+1} V^{*} \otimes V
$$

where $\mu$ is multiplication.

It will be convenient to express $\rho$ using the basis $\left\{x_{i}\right\}$ for $V^{*}$ so write

$$
\rho(X)=\sum \rho_{i}(X) \otimes \frac{\partial}{\partial x_{i}} .
$$

We have a composite map

$$
\mathfrak{g} \otimes V \stackrel{\rho \otimes \mathrm{id}}{\longrightarrow} \operatorname{Hom}(V, V) \otimes V \stackrel{c}{\rightarrow} V
$$

where $c$ is the contraction $c(\varphi \otimes v)=\varphi(v)$. Let $\beta: V^{*} \rightarrow \operatorname{Hom}\left(\mathfrak{g}, V^{*}\right)$ be the dual, i.e $\beta(\psi)(X)=\psi \circ \rho(X)$.

The map $d^{3}: \Omega_{S} \simeq S \otimes V^{*} \rightarrow S \otimes \mathfrak{g}^{*}$ on graded pieces is the composition

$$
S_{k} \otimes V^{*} \stackrel{\text { id } \otimes \beta}{\longrightarrow} S_{k} \otimes \operatorname{Sym}^{1} V^{*} \otimes \mathfrak{g}^{*} \stackrel{\mu \otimes \mathrm{id}}{\longrightarrow} S_{k+1} \otimes \mathfrak{g}^{*}
$$

where $\mu$ is the multiplication map. If we use the identification $S \otimes \mathfrak{g}^{*} \simeq \operatorname{Hom}(\mathfrak{g}, S)$ we get

$$
d^{3}(f \otimes \psi)(X)=f \sum_{j} \psi\left(\frac{\partial}{\partial x_{j}}\right) \rho_{j}(X) .
$$

In particular $d^{3}\left(d x_{i}\right)(X)=\rho_{i}(X)$.

\section{Cotangent cohomology of Plücker algebras}

Let $E$ be an $n$-dimensional vector space and $\mathbb{G}=\mathbb{G}(r, E)$ the Grassmannian of $r$-dimensional subspaces. Let $A$ be the homogeneous coordinate ring of $\mathbb{G}$ in the Plücker embedding. Fix an $r$-dimensional vector space $W$ and consider $V=\operatorname{Hom}(W, E)$ which we may think of as the space of $n \times r$ matrices. We have the natural action of $\mathrm{GL}(E) \times \mathrm{GL}(W)$ on $V$ and $V^{*}=E^{*} \otimes W$.

For this section set $G=\operatorname{SL}(W)$ and $S=\operatorname{Sym} V^{*}$ so that $A=S^{G}$. Set $d=\operatorname{dim} A=$ $(n-r) r+1$. We write

$$
S=k\left[x_{i j}: 1 \leq i \leq n, 1 \leq j \leq r\right]
$$

where after fixing basis $\left\{e_{i}\right\}$ for $E$ and $\left\{w_{j}\right\}$ for $W$ we have $x_{i j}=e_{i}^{*} \otimes w_{j}$.

Set $U^{*}=\bigwedge^{r} E^{*} \otimes \bigwedge^{r} W \subset \operatorname{Sym}^{r}\left(E^{*} \otimes W\right)$. Then a basis for $U^{*}$ form the generators of $J$, the ideal of maximal $r \times r$ minors in a general $n \times r$ matrix and they generate the algebra $A=S^{G}$. If $P=\operatorname{Sym} U^{*}$ then the kernel $I$ of the surjection $P \rightarrow A$ is generated by the quadratic Plücker relations.

Combining Proposition 2.3 with Proposition 3.4, Theorem 3.6 and Proposition 3.8, which are proven below, we get the following theorem 
Theorem 3.1. Assume $A$ is the Plücker algebra for a Grassmannian $\mathbb{G}(r, n)$ different from $\mathbb{G}(2,4)$. Then $T_{A}^{i}=0$ for $1 \leq i \leq d-1=r(n-r)$ and $T_{A}^{d}=0$ if and only if $r=2$ or $r=n-2$.

Remark. If $r \neq 2$ and $r \neq n-2$ then $T_{A}^{d}$ is concentrated in degree 2, see Theorem 3.6 below.

The result is sharp, i.e. we cannot expect $T_{A}^{d+1} \neq 0$ as seen in this example.

Example 3.2. Let $A$ be the Plücker algebra for $\mathbb{G}(2,6)$ of dimension 9 . Let $p_{i j}, 1 \leq i<j \leq 6$ be the Plücker coordinates. The ideal generated by

$$
p_{12}, p_{23}, p_{34}, p_{45}, p_{56}, p_{16}, p_{14}+p_{34}+p_{26}, p_{24}+p_{15}+p_{36}
$$

defines a codimension 8 complete intersection ideal in $A$. Let $B$ be the coordinate ring of this curve. A Macaulay2 computation shows that $\operatorname{dim} T_{B}^{2}=1$. By $[\mathrm{BC} 91,1.4 .2]$ this implies that $T_{A}^{10} \neq 0$.

3.1. About $H_{\mathfrak{m}}^{i}\left(\operatorname{Der}_{k}(A)\right)$. Let

$$
0 \rightarrow \mathcal{R} \rightarrow \mathcal{O}_{\mathbb{G}} \otimes E \rightarrow \mathcal{Q} \rightarrow 0
$$

be the tautological sequence on $\mathbb{G}$. Recall that $\Theta_{\mathbb{G}} \simeq \mathcal{R}^{\vee} \otimes_{\mathcal{O}_{\mathbb{G}}} \mathcal{Q}$ and that $\mathcal{O}_{\mathbb{G}}(m) \simeq$ $\left(\wedge^{n-r} \mathcal{Q}\right)^{\otimes m}$.

Lemma 3.3. There are isomorphisms of $\mathrm{SL}(E)$-modules

$$
H^{i}\left(\mathbb{G}, \mathcal{O}_{\mathbb{G}}(m)\right) \simeq \begin{cases}\mathbb{S}_{\left(m^{n-r}\right)} E & \text { if } i=0 \text { and } m \geq 0 \\ \mathbb{S}_{\left((-m-n)^{r}\right)} E & \text { if } i=r(n-r) \text { and } m \leq-n \\ 0 & \text { for all other values of } i \text { and } m\end{cases}
$$

and if $(r, n) \neq(2,4)$ then

$$
H^{i}\left(\mathbb{G}, \Theta_{\mathbb{G}}(m)\right) \simeq \begin{cases}\mathbb{S}_{\left(m+1, m^{n-r-1}, 0^{r-1},-1\right)} E & \text { if } i=0 \text { and } m \geq 0 \\ \mathbb{S}_{(0)} E & \text { if } i=r(n-r)-1 \text { and } m=-n \\ \mathbb{S}_{\left((-m-n)^{r-1},-m-n-1,1\right)} E & \text { if } i=r(n-r) \text { and } m \leq-n-2 \\ 0 & \text { for all other values of } i \text { and } m .\end{cases}
$$

Proof. We use Bott's theorem as described in Theorem 1.1. We only give the calculation for

$$
\Theta_{\mathbb{G}}(m) \simeq \mathbb{S}_{\left(m+1, m^{n-r-1}\right)} \mathcal{Q} \otimes \mathbb{S}_{\left(0^{r-1},-1\right)} \mathcal{R}
$$

Let $\lambda=\left(m+1, m^{n-r-1}, 0^{r-1},-1\right)$. If $\delta=(n-1, \ldots, 0)$ then

$$
\lambda+\delta=(m+n, m+n-2, \ldots, m+r, r-1, \ldots, 1,-1)
$$

cannot have repeated entries if $m>-1, m<-n-1$ or $m=-n$. On the other hand one can easily check that if if $m=-n-1$ or $-n+1 \leq m \leq-n+r-1$ then $m+n$ is repeated. If $-n+r+1 \leq m \leq-1$ then $m=-n+r+k$ with $1 \leq k \leq n-r-1$ so $m+n-2 \geq m+n-(k+1) \geq m+r$. Thus $m+n-(k+1)=r-1$ is repeated. If finally $m=-n+r$ assume first that $r \geq 3$. Then $m+n-2=r-2 \geq 1$ so it is repeated. If $r=2$ and $n \geq 5$ then $n-3 \geq r$ so $m+(n-3)=-1$ is repeated. We conclude that if $(r, n) \neq(2,4)$ then $H^{i}\left(\mathbb{G}, \Theta_{\mathbb{G}}(m)\right)=0$ for all values of $i$ if and only if $-n+1 \leq m \leq-1$ or $m=-n-1$.

If $m \geq 0$ then $\lambda+\delta$ is non-decreasing so $H^{0}\left(\mathbb{G}, \Theta_{\mathbb{G}}(m)\right) \simeq \mathbb{S}_{\lambda} E$ and all other cohomology vanishes. If $m \leq-n-2$ then $\lambda+\delta$ needs $r(n-r)$ adjacent transpositions to become the non-decreasing

$$
(r-1, r-2, \ldots, 1,-1, m+n, m+n-2, \ldots, m+r) .
$$


Subtracting $\delta$ we get

$$
\left((r-n)^{r-1}, r-1-n, m+r+1,(m+r)^{n-r-1}\right)
$$

so the only non-zero cohomology is $H^{r(n-r)}\left(\mathbb{G}, \Theta_{\mathbb{G}}(m)\right) \simeq \mathbb{S}_{\left((-m-n)^{r-1},-m-n-1,1\right)} E$ as $\operatorname{SL}(E)$ modules. If $m=-n$ then $\lambda+\delta$ needs $r(n-r)-1$ adjacent transpositions to become

$$
(r-1, \ldots, 1,0,-1,-2, \ldots,-n+r) .
$$

Subtracting $\delta$ we get $\left((r-n)^{n}\right)$ so the only non-zero cohomology is $H^{r(n-r)-1}\left(\mathbb{G}, \Theta_{\mathbb{G}}(-n)\right) \simeq$ $\mathbb{S}_{(0)} E \simeq k$ as $\mathrm{SL}(E)$-modules.

Remark. If we do the above calculation for $\Theta_{\mathbb{G}}(m)$ on $\mathbb{G}(2,4)$ we get

$$
\lambda+\delta=(m+4, m+2,1,-1)
$$

which has repeated entries iff $m$ equals $-1,-3$ or -5 . Thus in addition to the cohomology described in the lemma, we must check when $m=-2$. Then $\lambda+\delta$ needs one adjacent transposition to become $(2,1,0,-1)$ and subtracting $\delta$ we get $(-1,-1,-1,-1)$. Thus the isomorphism $H^{1}(\Theta(-2)) \simeq k$ corresponds to $\left(T_{A}^{1}\right)_{-2} \simeq k$.

Proposition 3.4. If $A$ is the Plücker algebra for $\mathbb{G}(r, n)$ which is not $\mathbb{G}(2,4)$, then

$$
H_{\mathfrak{m}}^{i}\left(\operatorname{Der}_{k}(A)\right)=0
$$

for $0 \leq i \leq d-1$

Proof. Since $\operatorname{depth}_{\mathfrak{m}} A \geq 2$, the module $H_{\mathfrak{m}}^{i}\left(\operatorname{Der}_{k}(A)\right)=0$ for $i=0,1$. The vanishing of $H_{\mathfrak{m}}^{i}\left(\operatorname{Der}_{k}(A)\right)$ for $i=2, \ldots, d-2$ follows from the sequence (2.1), Lemma 2.4 and Lemma 3.3.

To show that $H_{\mathfrak{m}}^{d-1}\left(\operatorname{Der}_{k}(A)\right)=0$ we must show that the connecting map

$$
H^{d-2}\left(\mathbb{G}, \Theta_{\mathbb{G}}(-n)\right) \rightarrow H^{d-1}\left(\mathbb{G}, \mathcal{O}_{\mathbb{G}}(-n)\right)
$$

is injective. Note that $\omega_{\mathbb{G}}=\mathcal{O}_{\mathbb{G}}(-n)$. Now we know from Proposition 2.6 that $(2.1)$ is the Atiyah extension, so by Serre duality this will follow if the connecting map $H^{0}\left(\mathbb{G}, \mathcal{O}_{\mathbb{G}}\right) \rightarrow$ $H^{1}\left(\mathbb{G}, \Omega_{\mathbb{G}}\right)$ from $e_{L}$ is an isomorphism. This is the map

$$
\operatorname{Hom}_{\mathbb{G}}\left(\mathcal{O}_{\mathbb{G}}, \mathcal{O}_{\mathbb{G}}\right) \stackrel{\gamma}{\rightarrow} \operatorname{Ext}_{\mathbb{G}}^{1}\left(\mathcal{O}_{\mathbb{G}}, \Omega_{\mathbb{G}}\right)
$$

from the long exact Ext-sequence of $e_{L}$. Recall that if

$$
e: 0 \rightarrow A \rightarrow B \rightarrow C \rightarrow 0
$$

is an exact sequence then the induced map $\operatorname{Hom}(M, C) \rightarrow \operatorname{Ext}^{1}(M, A)$ sends $\varphi$ to the class of the pullback over $\varphi$ of $e$. Thus $\gamma(\mathrm{id})$ is the class of $e_{L}$ and $\gamma$ is an isomorphism.

3.2. About $H_{\mathfrak{m}}^{0}\left(\Omega_{A}\right)$ and $H_{\mathfrak{m}}^{1}\left(\Omega_{A}\right)$. We now compute $H_{\mathfrak{m}}^{0}\left(\Omega_{A}\right)$ and $H_{\mathfrak{m}}^{1}\left(\Omega_{A}\right)$ using the complex

$$
C^{\bullet}: \quad(S \otimes F)^{G} \stackrel{d^{1}}{\longrightarrow}\left(S \otimes U^{*}\right)^{G} \stackrel{d^{2}}{\rightarrow}\left(\Omega_{S}\right)^{G} \stackrel{d^{3}}{\rightarrow}\left(S \otimes \mathfrak{g}^{*}\right)^{G}
$$

of Proposition 2.8 and the $\mathrm{GL}(E) \times \mathrm{GL}(W)$ action on everything. Let us first identify the representations corresponding to the modules involved.

If we use the $P$-grading on $S^{G}$ we have

$$
S^{G}=\bigoplus_{m \geq 0} S_{m}^{G} \simeq \bigoplus_{m \geq 0} \mathbb{S}_{\left(m^{r}\right)} E^{*}
$$


The ideal $I$ generated by the Plücker relations in $P_{2}$ is generated by

$$
F \simeq \bigoplus_{\substack{2 \leq i \leq \min (r, n-r) \\ i \text { even }}} \mathbb{S}_{\left(2^{r-i}, 1^{2 i}\right)} E^{*}
$$

(see e.g. [FH91, Exercise 15.43]). Thus the graded pieces of $(S \otimes F)^{G}=S^{G} \otimes F$ are

$$
\bigoplus_{\substack{2 \leq i \leq \min (r, n-r) \\ i \text { even }}} \mathbb{S}_{\left(m^{r}\right)} E^{*} \otimes \mathbb{S}_{\left(2^{r-i}, 1^{2 i}\right)} E^{*}
$$

for each $m \geq 0$. We have the graded pieces of $\left(S \otimes U^{*}\right)^{G}=S^{G} \otimes U^{*}$ given as

$$
S_{m}^{G} \otimes U^{*} \simeq \mathbb{S}_{\left(m^{r}\right)} E^{*} \otimes \mathbb{S}_{\left(1^{r}\right)} E^{*} \simeq \bigoplus_{0 \leq i \leq \min (r, n-r)} \mathbb{S}_{\left((m+1)^{r-i}, m^{i}, 1^{i}\right)} E^{*}
$$

(see e.g. [FH91, $\S 6.1(6.9)]$ ).

Now $\Omega_{S} \simeq S \otimes V^{*}=S \otimes W \otimes E^{*}$ as $\mathrm{GL}(E) \times \mathrm{GL}(W)$ module. The $S$-graded pieces are

$$
\operatorname{Sym}^{k}\left(W \otimes E^{*}\right) \otimes W \otimes E^{*} \simeq \bigoplus_{\lambda \vdash k}\left(\mathbb{S}_{\lambda} E^{*} \otimes E^{*}\right) \otimes\left(\mathbb{S}_{\lambda} W \otimes W\right) .
$$

The only $\lambda$ for which $\mathbb{S}_{\lambda} W \otimes W$ contains an $\mathrm{SL}(W)$ invariant subspace are

$$
\lambda=\left((m+1)^{r-1}, m\right)
$$

in degree $k=(m+1) r-1$ for some $m \geq 0$. The invariant part is

$$
\begin{aligned}
\left(S_{(m+1) r-1} \otimes V^{*}\right)^{G} & \simeq \mathbb{S}_{\left((m+1)^{r-1}, m\right)} E^{*} \otimes E^{*} \\
& \simeq\left\{\begin{array}{l}
\mathbb{S}_{\left(m+2,(m+1)^{r-2}, m\right)} E^{*} \oplus \mathbb{S}_{\left((m+1)^{r}\right)} E^{*} \oplus \mathbb{S}_{\left((m+1)^{r-1}, m, 1\right)} E^{*} \text { if } m \geq 1 \\
\mathbb{S}_{\left(2,1^{r-2}\right)} E^{*} \oplus \mathbb{S}_{\left(1^{r}\right)} E^{*} \text { if } m=0
\end{array}\right.
\end{aligned}
$$

as $\mathrm{GL}(E)$ representation.

We identify $\mathfrak{g}^{*}=\mathfrak{s l}_{r}^{*} \simeq \mathbb{S}_{\left(2,1^{r-2}\right)} W \otimes \bigwedge^{r} W^{*}$ so

$$
S_{k} \otimes \mathfrak{g}^{*} \simeq \bigoplus_{\lambda \vdash k} \mathbb{S}_{\lambda} E^{*} \otimes\left(\mathbb{S}_{\lambda} W \otimes \mathbb{S}_{\left(2,1^{r-2}\right)} W \otimes \bigwedge^{r} W^{*}\right) .
$$

The only $\lambda$ where $\mathbb{S}_{\lambda} W \otimes \mathbb{S}_{\left(2,1^{r-2}\right)} W$ contains an $\mathrm{SL}(W)$ trivial representation are

$$
\lambda=\left(m+2,(m+1)^{r-2}, m\right)
$$

in degree $k=(m+1) r$ for $m \geq 0$. So the invariant part is

$$
\left(S_{(m+1) r} \otimes \mathfrak{s l}_{r}^{*}\right)^{G} \simeq \mathbb{S}_{\left(m+2,(m+1)^{r-2}, m\right)} E^{*}
$$

as $\mathrm{GL}(E)$ representation.

The map $d^{2}: \Omega_{P} \otimes_{P} S^{G} \rightarrow \Omega_{S}^{G}$ is induced by the Jacobian matrix of the generators of $S^{G}$, i.e. the $r \times r$ minors. It has therefore $S$-degree $r-1$. Let $d_{m}^{2}$ be the map on graded pieces $\left(S_{m r}\right)^{G} \otimes U^{*} \rightarrow\left(S_{(m+1) r-1} \otimes V^{*}\right)^{G}$. 
Lemma 3.5. If $m \geq 1$,

$$
\operatorname{Im} d_{m}^{2} \simeq \mathbb{S}_{\left((m+1)^{r}\right)} E^{*} \oplus \mathbb{S}_{\left((m+1)^{r-1}, m, 1\right)} E^{*}
$$

and

$$
\operatorname{Ker} d_{m}^{2} \simeq \bigoplus_{2 \leq i \leq \min (r, n-r)} \mathbb{S}_{\left((m+1)^{r-i}, m^{i}, 1^{i}\right)} E^{*}
$$

as $\mathrm{GL}(E)$ representations.

Proof. Comparing (3.2) and (3.3) we see that the second statement follows from the first and that we must show that the endomorphisms on $\mathbb{S}_{\left((m+1)^{r}\right)} E^{*}$ and $\mathbb{S}_{\left((m+1)^{r-1}, m, 1\right)} E^{*}$ induced by $d^{2}$ are isomorphisms. By Schur's Lemma it is enough that $d^{2}$ is non-zero on them. Let $u_{1}, u_{2} \in U^{*}$ be $u_{1}=\left|x_{i j}\right|$ for $1 \leq i, j \leq r$ and $u_{2}=\left|x_{i j}\right|$ for $i=1, \ldots, r-1, r+1$ and $1 \leq j \leq r$. Thus $u_{1} \mapsto e_{1}^{*} \wedge \cdots \wedge e_{r}^{*}$ and $u_{2} \mapsto e_{1}^{*} \wedge \cdots \wedge e_{r-1}^{*} \wedge e_{r+1}^{*}$ via $U^{*} \simeq \wedge^{r} E^{*}$.

The part $\mathbb{S}_{\left((m+1)^{r}\right)} E^{*} \simeq S_{m+1}^{G} \subset S_{m}^{G} \otimes U^{*}$ corresponds to $\left\{d f: f \in S_{m+1}^{G}\right\}$ and clearly $d^{2}$ is non-zero on this. Indeed, the image of the highest weight vector $u_{1}^{m} \otimes d u_{1}$ is clearly non-zero. It is easily seen that $u_{1}^{m} \otimes d u_{2}$ is a weight vector for the highest weight $\left((m+1)^{r-1}, m, 1\right)$, so $u_{1}^{m} \otimes d u_{2}$ is in the $\mathbb{S}_{\left((m+1)^{r-1}, m, 1\right)} E^{*}$ part and does not map to 0 .

Theorem 3.6. If $A$ is the Plücker algebra for $\mathbb{G}(r, E)$ with $\operatorname{dim} E=n$, then $H_{\mathfrak{m}}^{0}\left(\Omega_{A}\right)$ vanishes if and only if $r=2$ or $r=n-2$. If $r \neq 2$ and $r \neq n-2$ then $H_{\mathfrak{m}}^{0}\left(\Omega_{A}\right)$ is concentrated in degree 2 and

$$
H_{\mathfrak{m}}^{0}\left(\Omega_{A}\right)_{2} \simeq \bigoplus_{\substack{3 \leq i \leq \min (r, n-r) \\ i \text { odd }}} \mathbb{S}_{\left(2^{r-i}, 1^{2 i}\right)} E^{*}
$$

and is therefore the kernel of the projection $\wedge^{2}\left(\wedge^{r} E^{*}\right) \rightarrow \mathbb{S}_{\left(2^{r-1}, 1^{2}\right)} E^{*}$.

Proof. Since the Plücker relations are in degree $2, d^{1}$ in the $P$-grading take $S_{m}^{G} \otimes F$ to $S_{m+1}^{G} \otimes$ $U^{*}$. If $m=0$ we get a map to $\operatorname{Ker} d_{1}^{2}$, i.e. from (3.1) and Lemma 3.5 a map

$$
\bigoplus_{\substack{2 \leq i \leq \min (r, n-r) \\ i \text { even }}} \mathbb{S}_{\left(2^{r-i}, 1^{2 i}\right)} E^{*} \rightarrow \bigoplus_{2 \leq i \leq \min (r, n-r)} \mathbb{S}_{\left(2^{r-i}, 1^{2 i}\right)} E^{*}
$$

which cannot be surjective unless $r$ or $n-r$ equals 2. The map is $f \mapsto d f$ which cannot be 0 on the generators of $I$, so by Schur's Lemma (3.5) is injective. Thus $H_{\mathfrak{m}}^{0}\left(\Omega_{A}\right)_{2}=0$ only for $r=2$ or $n-r=2$. Moreover

$$
\wedge^{2}\left(\wedge^{r} E^{*}\right) \simeq \bigoplus_{\substack{1 \leq i \leq \min (r, n-r) \\ i \text { odd }}} \mathbb{S}_{\left(2^{r-i}, 1^{2 i}\right)} E^{*}
$$

(see e.g. [FH91, Exercise 15.32]), so if $r$ and $n-r$ do not equal 2 then $H_{\mathfrak{m}}^{0}\left(\Omega_{A}\right)_{2}$ is isomorphic to the kernel of the projection $\wedge^{2}\left(\wedge^{r} E^{*}\right) \rightarrow \mathbb{S}_{\left(2^{r-1}, 1^{2}\right)} E^{*}$.

On the other hand we claim that when $m \geq 1$ the $\operatorname{map} S_{m}^{G} \otimes F \rightarrow \operatorname{Ker} d_{m+1}^{2}$ is surjective. We first check that the $\mathbb{S}_{\left((m+2)^{r-i},(m+1)^{i}, 1^{i}\right)} E^{*}$ for $2 \leq i \leq \min (r, n-r)$ all appear as summands in

$$
S_{m}^{G} \otimes F \simeq \bigoplus_{\substack{2 \leq i \leq \min (r, n-r) \\ i \text { even }}} \mathbb{S}_{\left(m^{r}\right)} E^{*} \otimes \mathbb{S}_{\left(2^{r-i}, 1^{2 i}\right)} E^{*}
$$


Indeed, if $i$ is even and $2 \leq i \leq \min (r, n-r)$ then an application of the Littlewood-Richardson rule shows that both

$$
\mathbb{S}_{\left((m+2)^{r-i},(m+1)^{i}, 1^{i}\right)} E^{*} \quad \text { and } \quad \mathbb{S}_{\left((m+2)^{r-(i+1)},(m+1)^{i+1}, 1^{i+1}\right)} E^{*}
$$

appear in the decomposition of $\mathbb{S}_{\left(m^{r}\right)} E^{*} \otimes \mathbb{S}_{\left(2^{r-i}, 1^{2 i}\right)} E^{*}$.

We must now show that the induced endomorphisms of the $\mathbb{S}_{\left((m+1)^{r-i}, m^{i}, 1^{i}\right)} E^{*}$ are isomorphisms. We do this by induction on $m$. The map $S_{m}^{G} \otimes F \rightarrow S_{m+1}^{G} \otimes U^{*}$ factors through $\left(I / I^{2}\right)_{m+2}$. Let $u_{0}$ be a Plücker coordinate and assume $f \in\left(I / I^{2}\right)_{m}$ with $d f \neq 0$ in $S^{G} \otimes U^{*}$. Then $d\left(u_{0} f\right)=u_{0} d f \neq 0$ in $S^{G} \otimes U^{*}$.

If $m=1$ let $f$ be a Plücker relation in $\mathbb{S}_{\left(2^{r-i}, 1^{2 i}\right)} E^{*}$ with $i$ even. Let $u_{0}$ correspond to $e_{1}^{*} \wedge \cdots \wedge e_{r}^{*}$ and $u_{1}$ correspond to $e_{1}^{*} \wedge \cdots \wedge \widehat{e_{r-i}^{*}} \wedge \cdots \wedge e_{r+1}^{*}$. Then $u_{0} \otimes f \in \mathbb{S}_{\left(3^{r-i}, 2^{i}, 1^{i}\right)} E^{*} \subset S_{1}^{G} \otimes F$ and $u_{1} \otimes f \in \mathbb{S}_{\left(3^{r-(i+1)}, 2^{i+1}, 1^{i+1}\right)} E^{*}$ and by the above they do not map to 0 . Now assume the maps are isomorphisms up to degree $m$. Let $f \in\left(I / I^{2}\right)_{m+2}$ be the image of something in $\mathbb{S}_{\left((m+2)^{r-i},(m+1)^{i}, 1^{i}\right)} E^{*}$. Then $u_{0} f$ is the image of something in $\mathbb{S}_{\left((m+3)^{r-i},(m+2)^{i}, 1^{i}\right)} E^{*}$ and by the above does not map to 0 .

Remark. The statement about $H_{\mathfrak{m}}^{0}\left(\Omega_{A}\right)_{2}$ follows for more general reasons from the fact that it is the kernel of the Gaussian map $\wedge^{2} H^{0}(X, L) \rightarrow H^{0}\left(X, \Omega_{X} \otimes L^{2}\right)$ for $L=\mathcal{O}_{X}(1)$ ([Wah97, Propositions 1.4 and 1.8]). In the same paper, see [Wah97, Problem 2.7], Wahl asks if, given a very ample line bundle on a complex homogeneous space $G / P$, the corresponding projective embedding in $\mathbb{P}^{N}$ has the property

$$
H^{1}\left(\mathbb{P}^{N}, \mathcal{I}^{2}(k)\right)=0, \text { for } k \neq 2 ?
$$

Here $\mathcal{I}$ is the ideal sheaf for the embedding. In our case $H_{\mathfrak{m}}^{0}\left(\Omega_{A}\right)_{k} \simeq H^{1}\left(\mathcal{I}^{2}(k)\right)$ ([Wah97, Propositions 1.4]) so our result on the vanishing of $H_{\mathfrak{m}}^{0}\left(\Omega_{A}\right)_{k}$ for $k \neq 2$ yields an affirmative answer in the case $G / P$ is a Grassmannian in the Plücker embedding.

The map $d^{3}: \Omega_{S}^{G} \simeq S \otimes V^{*} \rightarrow\left(S \otimes \mathfrak{s l}_{r}^{*}\right)^{G}$ on graded pieces is

$$
d_{m}^{3}:\left(S_{m r-1} \otimes V^{*}\right)^{G} \rightarrow\left(S_{m r} \otimes \mathfrak{s l}_{r}^{*}\right)^{G}
$$

for $m \geq 1$. To continue we will need SL(W)-invariants in $\Omega_{S}$. To make such, take an $r \times r$ submatrix of $\left(x_{i j}\right)$ and replace one of the rows with the tuple $\left(d x_{p, 1}, d x_{p, 2}, \ldots, d x_{p, r}\right)$. Now take the determinant to get an $\mathrm{SL}(W)$-invariant differential form. The special invariant form

$$
\delta=\left|\begin{array}{cccc}
x_{1,1} & x_{1,2} & \ldots & x_{1, r} \\
x_{2,1} & x_{2,2} & \ldots & x_{2, r} \\
\vdots & \vdots & \ddots & \vdots \\
x_{r-1,1} & x_{r-1,2} & \ldots & x_{r-1, r} \\
d x_{1,1} & d x_{1,2} & \ldots & d x_{1, r}
\end{array}\right|
$$

is a weight vector for the $\mathrm{GL}(E)$ action with weight $\left(2,1^{r-2}, 0^{n-r+1}\right)$. If $u=\left|x_{i j}\right|$ for $1 \leq$ $i, j \leq r$ then the invariant form $u^{m-1} \delta$ is a weight vector for $\left(m+1, m^{r-2}, m-1,0^{n-r}\right)$.

Lemma 3.7. As $\mathrm{GL}(E)$ representation $\operatorname{Ker} d_{m}^{3} \simeq \mathbb{S}_{\left(m^{r}\right)} E^{*} \oplus \mathbb{S}_{\left(m^{r-1}, m-1,1\right)} E^{*}$ for $m \geq 2$ and $\operatorname{Ker} d_{1}^{3} \simeq \wedge^{r} E^{*}$.

Proof. From (3.3) and (3.4) we must show that the endomorphism on $\mathbb{S}_{\left(m+1, m^{r-2}, m-1\right)} E^{*}$ induced by $d^{3}$ is non-zero. To do this it is enough by Schur's Lemma to show that $d^{3}\left(u^{m-1} \delta\right) \neq$ 
0 , which by linearity is the same as $d^{3}(\delta) \neq 0$. From (2.7) we get for $X \in \mathfrak{s l}_{r}$ that $d^{3}(\delta)(X)$ is the determinant

$$
\left|\begin{array}{cccc}
x_{1,1} & x_{1,2} & \ldots & x_{1, r} \\
x_{2,1} & x_{2,2} & \ldots & x_{2, r} \\
\vdots & \vdots & \ddots & \vdots \\
x_{r-1,1} & x_{r-1,2} & \ldots & x_{r-1, r} \\
\rho_{1,1}(X) & \rho_{1,2}(X) & \ldots & \rho_{1, r}(X)
\end{array}\right|
$$

so let $X=w_{r}^{*} \otimes w_{1}$. Then $\rho(X)=\sum_{i} x_{i, 1} \frac{\partial}{\partial x_{i, r}}$ and the last row in the determinant is $\left(0, \ldots, 0, x_{1,1}\right)$. Thus $d^{3}(\delta)(X) \neq 0$.

Proposition 3.8. If $A$ is the Plücker algebra for $\mathbb{G}(r, E)$ with $\operatorname{dim} E=n$, then $H_{\mathfrak{m}}^{1}\left(\Omega_{A}\right)=0$.

Proof. From Lemma 3.5 and Lemma 3.7 we get $\operatorname{Ker} d_{m+1}^{3}=\operatorname{Im} d_{m}^{2}$ for $m \geq 1$ and clearly $\operatorname{Im} d_{0}^{2} \simeq \wedge^{r} E^{*} \simeq \operatorname{Ker} d_{1}^{3}$. Thus $\operatorname{Ker} d^{3}=\operatorname{Im} d^{2}$.

\section{Cotangent Cohomology for isotropic Grassmannians}

In this section, we partially extend our vanishing results for Plücker algebras to the setting of isotropic Grassmannians. Fix $n \geq 2,1 \leq r \leq n$ and let $\mathbb{L} \mathbb{G}(r, 2 n), \mathbb{O} \mathbb{G}(r, 2 n)$, and $\mathbb{O} \mathbb{G}(r, 2 n+1)$ respectively denote the symplectic/orthogonal Grassmannians of isotropic $r$ planes in a $2 n$ (or $2 n+1)$-dimensional vector space. To avoid degenerate cases, and those coinciding with classical Grassmannians, we will make the following assumptions throughout:

(1) For $\mathbb{L} \mathbb{G}(r, 2 n), r>1$ and $n \geq 2$;

(2) For $\mathbb{O G}(r, 2 n), n \geq 4$ and $r \neq n-1$;

(3) For $\mathbb{O G}(r, 2 n+1), r \geq 1$ and $n \geq 2$.

Note that $\mathbb{O} \mathbb{G}(n, 2 n)$ designates one of the two connected components of the Grassmannian of isotropic $n$ planes in a $2 n$-dimensional vector space. We consider each such Grassmannian in its Plücker embedding, and denoting its coordinate ring by $A$ and Serre's twisting sheaf by $\mathcal{O}(1)$. Set $d=\operatorname{dim} A=\operatorname{dim} X+1$, where $X$ is the appropriate isotropic Grassmannian. Our main result is

Theorem 4.1. Assume $A$ is the coordinate ring for an isotropic Grassmannian $X$ different from $\mathbb{L} \mathbb{G}(3,6)$. Then $T_{A}^{i}=0$ for $2 \leq i \leq d-3$, and $T_{A}^{d-2}=0$ if and only if $X$ is either $\mathbb{L} \mathbb{G}(n-1,2 n)$ or $\mathbb{O} \mathbb{G}(n, 2 n+1)$. Furthermore, $T_{A}^{1}=0$ as long as $X$ is not an isotropic Grassmannian of 1 or 2 -planes, or $\mathbb{O} \mathbb{G}(4,8)$.

Proof. Combine Proposition 2.5 with Theorems 4.2 and 4.3 below.

In addition to being useful for proving Theorem 4.1, the following cohomology vanishing is interesting in its own right:

Theorem 4.2. Let $X$ be $\mathbb{L} \mathbb{G}(r, 2 n), \mathbb{O} \mathbb{G}(r, 2 n)$, or $\mathbb{O} \mathbb{G}(r, 2 n+1)$. The cohomology

$$
H^{i}\left(X, \Theta_{X}(m)\right)
$$

vanishes for all $m \in \mathbb{Z}$ and $2 \leq i \leq d-3$, except for $X=\mathbb{L} \mathbb{G}(3,6)$. The cohomology

$$
H^{1}\left(X, \Theta_{X}(m)\right)
$$


vanishes for all $m \in \mathbb{Z}$ if $r \neq 1,2$ and $X \neq \mathbb{O} \mathbb{G}(4,8)$. Conversely, this cohomology group is non-zero for some $m \in \mathbb{Z}$ if $X$ is $\mathbb{L} \mathbb{G}(2,2 n)$ for $n \neq 3, \mathbb{O} \mathbb{G}(1,2 n), \mathbb{O} \mathbb{G}(4,4)$, or $\mathbb{O} \mathbb{G}(1,2 n+1)$. Finally, the cohomology

$$
H^{d-2}\left(X, \Theta_{X}(m)\right)
$$

vanishes for all $m \in \mathbb{Z}$ if and only if $X$ is either $\mathbb{L} \mathbb{G}(n-1,2 n)$ or $\mathbb{O} \mathbb{G}(n, 2 n+1)$.

Theorem 4.3. For $X=\mathbb{L} \mathbb{G}(r, 2 n), X=\mathbb{O} \mathbb{G}(r, 2 n)$, or $X=\mathbb{O} \mathbb{G}(r, 2 n+1)$, the cohomology $H^{i}\left(X, \mathcal{O}_{X}(m)\right)$ vanishes for all $m \in \mathbb{Z}$ for all $1 \leq i \leq d-2$.

Let $\mathcal{R}$ be the tautological bundle on $X$, and $\mathcal{R}^{\vee}$ the orthogonal complement. Then there are exact sequences

$$
0 \rightarrow \mathcal{R}^{*} \otimes\left(\mathcal{R}^{\vee} / \mathcal{R}\right) \rightarrow \Theta_{X} \rightarrow D_{2}\left(\mathcal{R}^{*}\right) \rightarrow 0
$$

when $X$ is a symplectic Grassmannian, and

$$
0 \rightarrow \mathcal{R}^{*} \otimes\left(\mathcal{R}^{\vee} / \mathcal{R}\right) \rightarrow \Theta_{X} \rightarrow \bigwedge^{2} \mathcal{R}^{*} \rightarrow 0
$$

when $X$ is an orthogonal Grassmannian, see [Wey03, Ch. 4 Ex. $9 \& 10]$. Here $D_{2}\left(\mathcal{R}^{*}\right)=$ $\left(\operatorname{Sym}^{2} \mathcal{R}\right)^{*}$ is the second divided power. We will prove Theorem 4.2 by considering the long exact sequence of cohomology of twists of these short exact sequences. For this, we need the following vanishing results for the left and right terms in the above sequences:

Lemma 4.4. The cohomology

$$
H^{i}\left(\mathbb{L} \mathbb{G}(r, 2 n), D_{2}\left(\mathcal{R}^{*}\right)(m)\right)
$$

vanishes for all $m \in \mathbb{Z}$ and $2 \leq i \leq d-3$, except for $\mathbb{L} \mathbb{G}(3,6)$. The cohomology

$$
H^{1}\left(\mathbb{L} \mathbb{G}(r, 2 n), D_{2}\left(\mathcal{R}^{*}\right)(m)\right)
$$

vanishes for all $m \in \mathbb{Z}$ if and only if $(r, n) \neq(2,2)$. Finally, the cohomology

$$
H^{d-2}\left(\mathbb{L} \mathbb{G}(r, 2 n), D_{2}\left(\mathcal{R}^{*}\right)(m)\right)
$$

vanishes for all $m \in \mathbb{Z}$ if and only if $r \neq n$.

Lemma 4.5. Let $X$ be $\mathbb{O G}(r, 2 n)$ or $\mathbb{O} \mathbb{G}(r, 2 n+1)$. The cohomology

$$
H^{i}\left(X,\left(\bigwedge^{2} \mathcal{R}^{*}\right)(m)\right)
$$

vanishes for all $m \in \mathbb{Z}$ and $2 \leq i \leq d-3$. The cohomology

$$
H^{1}\left(X,\left(\bigwedge^{2} \mathcal{R}^{*}\right)(m)\right)
$$

vanishes for all $m \in \mathbb{Z}$ if and only if $X$ is not equal to $O G(1,2 n), O G(4,4)$, or $O G(1,2 n+1)$. Finally, the cohomology

$$
H^{d-2}\left(X,\left(\bigwedge^{2} \mathcal{R}^{*}\right)(m)\right)
$$

vanishes for all $m \in \mathbb{Z}$ if and only if $X$ is not equal to $\mathbb{O} \mathbb{G}(1,2 n), \mathbb{O G}(n, 2 n)$, or $\mathbb{O} \mathbb{G}(1,2 n+1)$. 


\begin{tabular}{|c|c|}
\hline \multicolumn{2}{|l|}{$\mathfrak{g}=\mathfrak{s p}_{n}$} \\
\hline$\alpha_{i}+\ldots+\alpha_{j}$ & $i \leq j \leq n$ \\
\hline $2 \alpha_{j}+\ldots+2 \alpha_{n-1}+\alpha_{n}$ & $j<n$ \\
\hline$\alpha_{i}+\ldots+\alpha_{j-1}+2 \alpha_{j}+\ldots+2 \alpha_{n-1}+\alpha_{n}$ & $i<j<n$ \\
\hline \multicolumn{2}{|l|}{$\mathfrak{g}=\mathfrak{s o}_{2 n}$} \\
\hline$\alpha_{j}$ & $j=n-1, n$ \\
\hline$\alpha_{i}+\ldots+\alpha_{j}$ & $i \leq j \leq n-2$ \\
\hline$\alpha_{i}+\ldots+\alpha_{n-2}+\alpha_{j}$ & $i \leq n-2, j=n-1, n$ \\
\hline$\alpha_{i}+\ldots+\alpha_{n}$ & $i \leq n-2$ \\
\hline$\alpha_{i}+\ldots+\alpha_{j-1}+2 \alpha_{j}+\ldots+2 \alpha_{n-2}+\alpha_{n-1}+\alpha_{n}$ & $i<j \leq n-2$ \\
\hline \multicolumn{2}{|l|}{$\mathfrak{g}=\mathfrak{s o}_{2 n+1}$} \\
\hline $\begin{array}{r}\alpha_{i}+\ldots+\alpha_{j} \\
\alpha_{i}+\ldots+\alpha_{j-1}+2 \alpha_{j}+\ldots+2 \alpha_{n}\end{array}$ & $\begin{array}{l}\leq j \leq n \\
<j \leq n\end{array}$ \\
\hline
\end{tabular}

TABLE 1. Positive roots of $\mathfrak{g}$

Lemma 4.6. Let $X$ be $\mathbb{L} \mathbb{G}(r, 2 n)$ or $\mathbb{O} \mathbb{G}(r, 2 n)$ with $r<n$, or $\mathbb{O} \mathbb{G}(r, 2 n+1)$. The cohomology

$$
H^{i}\left(X, \mathcal{R}^{*} \otimes\left(\mathcal{R}^{\vee} / \mathcal{R}\right)(m)\right)
$$

vanishes for all $m \in \mathbb{Z}$ and $2 \leq i \leq d-3$. The cohomology

$$
H^{1}\left(X, \mathcal{R}^{*} \otimes\left(\mathcal{R}^{\vee} / \mathcal{R}\right)(m)\right)
$$

vanishes for all $m \in \mathbb{Z}$ if and only if $X$ is not equal to $\mathbb{L} \mathbb{G}(2, n)$ for $n>3, \mathbb{O} \mathbb{G}(r, 2 n)$ for $r=1,2$, or $\mathbb{O} \mathbb{G}(r, 2 n+1)$ for $r=1,2$ with $r \neq n$. Finally, the cohomology

$$
H^{d-2}\left(X, \mathcal{R}^{*} \otimes\left(\mathcal{R}^{\vee} / \mathcal{R}\right)(m)\right)
$$

vanishes for all $m \in \mathbb{Z}$ if and only if $X$ equals $\mathbb{L} \mathbb{G}(n-1,2 n)$ or $\mathbb{O} \mathbb{G}(n, 2 n+1)$.

Proof of Lemmata 4.4, 4.5, and 4.6. We will prove these lemmata using Bott's theorem for isotropic Grassmannians [Wey03, 4.3.4, 4.3.7, \& 4.3.9]. First we need some notation. Let $\mathfrak{g}$ be one of the Lie algebras $\mathfrak{s p}_{n}, \mathfrak{s o}_{2 n}$, or $\mathfrak{s o}_{2 n+1}, \alpha_{1}, \ldots, \alpha_{n}$ its simple roots, and $\delta_{1}, \ldots, \delta_{n}$ the corresponding fundamental weights. We always assume that $n>1$. The positive roots of $\mathfrak{g}$ are exactly as listed in Table 1.

For any weight $\beta$ of type $A_{r-1}$ let $K_{\beta}$ be the corresponding Weyl functor, and for any weight $\mu$ of type $B_{n-r}, C_{n-r}$, or $D_{n-r}$ let $V_{\mu}\left(\mathcal{R}^{\vee} / \mathcal{R}\right)$ be the bundle defined fiberwise by the representation of weight $\mu$ with respect to the symplectic/orthogonal fibers of $\mathcal{R}^{\vee} / \mathcal{R}$. We then have

$$
\begin{aligned}
D_{2}\left(\mathcal{R}^{*}\right)=K_{\beta}(\mathcal{R}) & \text { for } & \beta=(0, \ldots, 0,-2) \\
2 & & \\
\bigwedge \mathcal{R}^{*}=K_{\beta}(\mathcal{R}) & \text { for } & \beta=(0, \ldots, 0,-1,-1) \\
\mathcal{R}^{*} \otimes\left(\mathcal{R}^{\vee} / \mathcal{R}\right)=K_{\beta}(\mathcal{R}) \otimes V_{\mu}\left(\mathcal{R}^{\vee} / \mathcal{R}\right) & \text { for } & \beta=(0, \ldots, 0,-1), \mu=(1,0, \ldots, 0) .
\end{aligned}
$$

By Bott's theorem [Wey03, 4.3.4, 4.3.7, \& 4.3.9], the $i$ th cohomology of the twist by $\mathcal{O}(m)$ of the above bundles is non-zero exactly when the weight $\gamma$ is non-singular of index $i$, where $\gamma$ 


\begin{tabular}{|c|c|c|c|c|}
\hline $\mathfrak{g}$ & $r, n$ & $\alpha(\gamma)$ for $\# \alpha_{r}=1$ & $\operatorname{Max} \alpha(\gamma)$ for $\# \alpha_{r}=2$ & Other $\alpha(\gamma)$ \\
\hline $\mathfrak{s p}_{n}$ & $1<r<n-1$ & $m+1, \ldots, m+n+(n-r)+1$ & $2 m+2 n+3$ & \\
\hline $\mathfrak{s p}_{n}$ & $1<r=n-1$ & $m+1, \ldots, m+n-1, m+n+1, m+n+2$ & $2 m+2 n+3$ & $2(m+n)$ \\
\hline $\mathfrak{s p}_{n}$ & $3<r=n$ & $m+1, \ldots, m+2 n, m+2 n+3$ & & \\
\hline $\mathfrak{s p}_{n}$ & $3=r=n$ & $m+1, m+2, m+3, m+5, m+6, m+9$ & & \\
\hline $\mathfrak{s p}_{n}$ & $2=r=n$ & $m+1, m+4, m+7$ & & \\
\hline
\end{tabular}

TABLE 2. $\alpha(\gamma)$ for $\gamma=2 \delta_{1}+m \delta_{r}+\sum \delta_{j}$

\begin{tabular}{lllll}
\hline $\mathfrak{g}$ & $r, n$ & $\alpha(\gamma)$ for $\# \alpha_{r}=1$ & Max $\alpha(\gamma)$ for \# $\alpha_{r}=2$ & Other $\alpha(\gamma)$ \\
\hline & & & \\
$\mathfrak{s o}_{2 n}$ & $2<r<n-1$ & $m+1, \ldots, m+n+(n-r)$ & $2(m+n)$ \\
$\mathfrak{s o}_{2 n}$ & $r \geq n-1, n>4$ & $m+1, \ldots, m+2 n-2, m+2 n$ & \\
$\mathfrak{s o}_{2 n}$ & $r=2, n \geq 4$ & $m+2, \ldots, m+2 n-2$ & $2(m+n)$ \\
$\mathfrak{s o}_{2 n}$ & $r=1, n \geq 4$ & $m+2, m+4, \ldots, m+2 n-2, m+2 n$ & \\
& & & \\
$\mathfrak{s o}_{2 n+1}$ & $2<r<n$ & $m+1, \ldots, m+n+(n-r)+2$ & $2(m+n+1)$ \\
$\mathfrak{s o}_{2 n+1}$ & $2=r<n$ & $m+2, \ldots, m+2 n$ & $2(m+n+1)$ \\
$\mathfrak{s o}_{2 n+1}$ & $2<r=n$ & $m+1 \ldots, m+n-2, m+n, m+n+2$ & $2(m+n+1)$ & $2(m+n-1)$ \\
$\mathfrak{s o}_{2 n+1}$ & $r=2, n=2$ & $m+2, m+4$ & $2(m+3)$ \\
$\mathfrak{s o}_{2 n+1}$ & $r=1$ & $m+2, m+4, \ldots, m+2 n, m+2 n+2$ & \\
& & & \\
\hline
\end{tabular}

TABLE 3. $\alpha(\gamma)$ for $\gamma=\delta_{1}+\delta_{2}+m \delta_{r}+\sum \delta_{j}$

is respectively

$$
\begin{aligned}
& \gamma=2 \delta_{1}+m \delta_{r}+\sum_{j=1}^{n} \delta_{j} \\
& \gamma=\delta_{1}+\delta_{2}+m \delta_{r}+\sum_{j=1}^{n} \delta_{j} ; \text { or } \\
& \gamma=\delta_{1}+m \delta_{r}+\delta_{r+1}+\sum_{j=1}^{n} \delta_{j} .
\end{aligned}
$$

Recall that the index of $\gamma$ is the number of positive roots $\alpha$ such that $\alpha(\gamma)<0$. Note that the only roots for which this can occur are those which involve $\alpha_{r}$, of which there are exactly $d-1$. Denote this set of $d-1$ roots by $S$. Furthermore, if $\alpha(\gamma)<0$ for any positive root $\alpha$, then $\alpha_{r}(\gamma)<0$. In Tables 2, 3, and 4, we list all values of $\alpha(\gamma)$ for those $\alpha \in S$ with $\alpha\left(\delta_{r}\right)=1$, the maximal value of $\alpha(\gamma)$ for those $\alpha \in S$ with $\alpha\left(\delta_{r}\right)=2$, along with (in some cases) further values of $\alpha(\gamma)$. These lists follow from Table 1 by inspection.

The claims of the lemmata now follow from Tables 2, 3, and 4. Indeed, suppose that $\gamma$ is non-singular of some strictly positive index $i$. Then the values of $\gamma(\alpha)$ cannot contain 0 , must contain $i$ negative values, and must contain $d-i-1$ positive values. Inspection of the tables leads to bounds on $i$. For example, consider the case $\mathfrak{g}=\mathfrak{s p}_{n}, 1<k=n-1$, and $\gamma=2 \delta_{1}+m \delta_{k}+\sum \delta_{j}$ (see Table 2). It follows that $m+1<0$, from which follows that $m+n-1<0$. If $m+n=0$, then $2(m+n)=0$ as well, which is impossible, since $\gamma$ is 


\begin{tabular}{|c|c|c|c|c|}
\hline $\mathfrak{g}$ & $r, n$ & $\alpha(\gamma)$ for $\# \alpha_{r}=1$ & $\operatorname{Max} \alpha(\gamma)$ for $\# \alpha_{r}=2$ & Other $\alpha(\gamma)$ \\
\hline $\mathfrak{s p}_{n}$ & $2<r<n-1$ & $m+1, \ldots, m+n+(n-r), m+n+(n-r)+2$ & $2 m+2 n+3$ & \\
\hline $\mathfrak{s p}_{n}$ & $2<r=n-1$ & $m+1, \ldots, m+n, m+n+2$ & $2(m+n+1)$ & \\
\hline $\mathfrak{s p}_{n}$ & $2=r<n-1$ & $m+1, m+3, \ldots, m+2 n-2, m+2 n$ & $2 m+2 n+3$ & \\
\hline $\mathfrak{s p}_{n}$ & $r=2, n=3$ & $m+1, m+3, m+5$ & $2(m+4)$ & $2(m+2)$ \\
\hline $\mathfrak{s o}_{2 n}$ & $2<r<n-1$ & $m+1, \ldots, m+n+(n-r)-1, m+n+(n-r)+1$ & $2(m+n)$ & \\
\hline $\mathfrak{s o}_{2 n}$ & $r=2, n \geq 4$ & $m+1, m+3, \ldots, m+2 n-3, m+2 n-1$ & $2(m+n)$ & \\
\hline $\mathfrak{s o}_{2 n}$ & $r=1, n \geq 4$ & $m+2, m+4, \ldots, m+2 n-2, m+2 n$ & & \\
\hline $\mathfrak{s o}_{2 n+1}$ & $2<r<n-1$ & $m+1, \ldots, m+n+(n-r)+1, m+n+(n-r)+3$ & $2(m+n+1)$ & \\
\hline $\mathfrak{s o}_{2 n+1}$ & $2<r=n-1$ & $m+1, \ldots, m+n+2, m+n+4$ & $2(m+n+1)$ & \\
\hline $\mathfrak{s o}_{2 n+1}$ & $2<r=n$ & $m+1, \ldots, m+n-1, m+n+1$ & $2 m+2 n$ & \\
\hline $\mathfrak{s o}_{2 n+1}$ & $2=r<n-1$ & $m+1, m+3, \ldots, m+2 n-1, m+2 n+1$ & $2(m+n+1)$ & \\
\hline $\mathfrak{s o}_{2 n+1}$ & $r=2, n=3$ & $m+1, m+3, m+5, m+7$ & $2(m+4)$ & \\
\hline $\mathfrak{s o}_{2 n+1}$ & $r=2, n=2$ & $m+1, m+3$ & $2(m+2)$ & \\
\hline $\mathfrak{s o}_{2 n+1}$ & $r=1$ & $m+2, m+4, \ldots, m+2 n, m+2 n+2$ & & \\
\hline
\end{tabular}

TABLE 4. $\alpha(\gamma)$ for $\gamma=\delta_{1}+m \delta_{r}+\delta_{r+1}+\sum \delta_{j}$

non-singular. So in fact, $m+n<0$, as are also $m+n+1$ and $m+n+2$. We thus conclude that in this case, $i=d-1$. All other cases are similarly straightforward.

Proof of Theorem 4.2. If $r=n$, then $\mathcal{R}^{\vee} / \mathcal{R}=0$, so $\Theta_{X}=D_{2}\left(\mathcal{R}^{*}\right)$ or $\Theta_{X}=\bigwedge^{2} \mathcal{R}^{*}$ and the claims follow directly from Lemmata 4.4 and 4.5. For $r<n$, we apply Lemmata 4.4, 4.5 and 4.6 to the long exact sequence of cohomology. For the claim regarding $H^{1}$ for $L G(2,2 n)$ with $n>3$, note that $H^{1}\left(L G(2,2 n), D_{2}\left(\mathcal{R}^{*}\right)(-2)\right)$ is non-vanishing, but $H^{0}\left(L G(2,2 n)\right.$, $\mathcal{R}^{*} \otimes$ $\left.\left.\left(\mathcal{R}^{\vee} / \mathcal{R}\right)\right)(-2)\right)=0$.

Proof of Theorem 4.3. By Bott's theorem, the $i$ th cohomology of $\mathcal{O}_{X}(m)$ vanishes unless the weight

$$
\gamma=m \delta_{r}+\sum_{j=1}^{n} \delta_{j}
$$

is non-singular of index $i$. The claim now follows from arguments similar to those used to prove the above lemmata.

\section{Deforming COMPlete intersections in CONES OVER Grassmannians}

Lemma 5.1. Let $A$ be a k-algebra, $I$ a complete intersection ideal in $A$ and $l$ the minimal number of generators for $I$. If $T_{A}^{i}=0$ for $1 \leq i \leq l+1$ then $T_{A}^{1}(A / I)=0$.

Proof. Let $B=A / I$. We have a long exact sequence

$$
\cdots \rightarrow T_{A}^{i}(I) \rightarrow T_{A}^{i} \rightarrow T_{A}^{i}(B) \rightarrow T_{A}^{i+1}(I) \rightarrow \cdots
$$

Let $F$ be a free $A$-module of rank equal to the number of generators of $I$ and consider the resolution of $I$ by the Koszul complex

$$
0 \rightarrow \bigwedge^{l} F \stackrel{d_{l}}{\rightarrow} \cdots \stackrel{d_{3}}{\rightarrow} \bigwedge^{2} F \stackrel{d_{2}}{\rightarrow} F \stackrel{d_{1}}{\rightarrow} I \rightarrow 0
$$


which we can split into short exact sequences

$$
0 \rightarrow I_{j} \rightarrow \bigwedge^{j} F \rightarrow I_{j-1} \rightarrow 0
$$

with $I_{0}:=I$ and $I_{j}:=\operatorname{ker} d_{j}$.

We show that $T_{A}^{p}\left(I_{j}\right)=0$ for $j+2 \geq p>1$ by induction on $j$. Indeed, $T_{A}^{p}\left(I_{l}\right)=0$ for all $p>1$ since $I_{l}=0$. Suppose that we have shown $T_{A}^{p}\left(I_{j}\right)=0$ for all $j+2 \geq p>1$. Consider any $p$ satisfying $j+1 \geq p>1$. Then $T_{A}^{p}\left(I_{j-1}\right)$ vanishes if $T_{A}^{p}\left(\bigwedge^{j} F\right)$ does. But $\bigwedge^{j} F$ is free, so $T_{A}^{p}=0$ implies $T_{A}^{p}\left(\bigwedge^{j} F\right)=0$. Thus since both $T_{A}^{1}$ and $T_{A}^{2}(I)$ vanish, we get $T_{A}^{1}(B)=0$ as desired.

Let $X=\operatorname{Proj} A \subseteq \mathbb{P}^{n}$. We say that $Y \subset X$ is a complete intersection in $X$ if $Y=\operatorname{Proj} B$ is of codimension $l$ in $X$ with $B=A /\left(f_{1}, \ldots, f_{l}\right)$ for $l$ homogeneous polynomials in $k\left[x_{0}, \ldots, x_{n}\right]$.

Proposition 5.2. Let $X=\operatorname{Proj} A \subseteq \mathbb{P}^{n}$ and assume $Y=\operatorname{Proj} B \subset X$ is a codimension $l$ complete intersection in $X$. Let $\mathfrak{m}$ be the irrelevant maximal ideal in $k\left[x_{0}, \ldots, x_{n}\right]$. If

(i) $\operatorname{depth}_{\mathfrak{m}} B \geq 3$

(ii) $H^{2}\left(Y, \mathcal{O}_{Y}\right)=0$

(iii) $T_{A}^{i}=0$ for $1 \leq i \leq l+1$

then any deformation of $Y$ is again a complete intersection in $X$.

Proof. The statement will follow if the forgetful map $\operatorname{Def}_{Y / X} \rightarrow \operatorname{Def}_{Y}$ from the local Hilbert functor of $Y$ in $X$ to the deformation functor of $Y$ is smooth. This follows if $T_{X}^{1}\left(\mathcal{O}_{Y}\right)=0$. Combine Lemma 4.2, Lemma 4.3 and Proposition 4.24 in [CK14] to see that the first two conditions guarantee a surjection $T_{A}^{1}(B)_{0} \rightarrow T_{X}^{1}\left(\mathcal{O}_{Y}\right)$. Thus the third assumption and Lemma 5.1 imply the result.

If $A$ is a graded $k$-algebra we write $A\left[x_{1}, \ldots, x_{m}\right]$ for the graded ring $A \otimes_{k} k\left[x_{1}, \ldots, x_{m}\right]$ where the degrees of the $x_{i}$ are 1 .

Corollary 5.3. Let $A$ be the Plücker algebra for $\mathbb{G}(r, n), d=\operatorname{dim} A=r(n-r)+1$ and $X=\operatorname{Proj} A\left[x_{1}, \ldots, x_{m}\right]$. If $Y$ is a complete intersection of codimension less thand in $X$ (and $\left.H^{2}\left(Y, \mathcal{O}_{Y}\right)=0\right)$ then any deformation of $Y$ is again a complete intersection in $X$.

Remark. Let $X$ be as above, and let $Y$ be a complete intersection of type $\left(a_{1}, \ldots, a_{k}\right)$ in $X$, where $m \leq k<d$, and $\sum a_{i}<n$. Then $Y$ is a (possibly singular) Fano variety. By the above corollary, any smoothing of $Y$ is again a complete intersection of type $\left(a_{1}, \ldots, a_{k}\right)$ in $X$.

\section{REFERENCES}

[And74] Michel André, Homologie des algèbres commutatives, Springer-Verlag, 1974.

[BC91] Kurt Behnke and Jan Arthur Christophersen, Hypersurface sections and obstructions (rational surface singularities), Compos. Math. 77 (1991), 233-268.

[CK14] Jan Arthur Christophersen and Jan O. Kleppe, Comparison theorems for deformation functors via invariant theory, arXiv:1209.3444v3 [math.AG], 2014.

[FH91] William Fulton and Joe Harris, Representation theory, Graduate Texts in Mathematics, vol. 129, Springer-Verlag, 1991.

[Gro05] Alexander Grothendieck, Cohomologie locale des faisceaux cohérents et théorèmes de Lefschetz locaux et globaux (SGA 2), Documents Mathématiques (Paris) [Mathematical Documents (Paris)], 4, Société Mathématique de France, Paris, 2005.

[Ill71] Luc Illusie, Complexe cotangent et déformations. I, Lecture Notes in Mathematics, Vol. 239, Springer-Verlag, Berlin, 1971. 
[Lau79] Olav Arnfinn Laudal, Formal moduli of algebraic structures, Lecture Notes in Mathematics, vol. 754, Springer-Verlag, 1979.

[RWW14] Claudiu Raicu, Jerzy Weyman, and Emily E. Witt, Local cohomology with support in ideals of maximal minors and sub-maximal Pfaffians, Adv. Math. 250 (2014), 596-610.

[Sch71] Michael Schlessinger, Rigidity of quotient singularities, Invent. Math. 14 (1971), 17-26.

[Sva75] Torgny Svanes, Some criteria for rigidity of noetherian rings, Math. Z. 144 (1975), 135-145.

[Wah97] Jonathan Wahl, On cohomology of the square of an ideal sheaf, J. Algebraic Geom. 6 (1997), 481511.

[Wey03] Jerzy Weyman, Cohomology of vector bundles and syzygies, Cambridge University Press, 2003. 